\title{
Double Exponential Integrability of Convolution Operators in Generalized Lorentz-Zygmund Spaces
}

\author{
David E. Edmunds, Petr Gurka \& Bohumír Opic
}

\begin{abstract}
This paper provides estimates for an appropriate norm of the convolution of a function in a Lorentz space with one in a generalized Lorentz-Zygmund space. As a corollary, it is shown that the Riesz potential of a function in an appropriate generalized Lorentz-Zygmund space satisfies a 'double exponential' integrability condition. The results extend those of Brézis-Wainger on the convolution of functions in Lorentz spaces which lead to exponential integrability.
\end{abstract}

1. Introduction. Let $\Omega$ be a bounded domain in $\mathbb{R}^{n}$. Trudinger $[\mathrm{T}]$ proved that the Sobolev space $W^{1, n}(\Omega)$ is continuously imbedded into the Orlicz space $L_{\Phi}(\Omega)$ with the Young function $\Phi(t):=\exp |t|^{n /(n-1)}-1$ provided that the boundary of $\Omega$ is sufficiently regular. This result is optimal (cf. [HMT]) in the sense that the function $\Phi$ cannot be replaced by any Young function $\Psi$ which increases strictly more rapidly than $\Phi$.

It is well-known that imbedding theorems for Sobolev spaces are closely related to results on the behaviour of the Riesz potential on Lebesgue spaces. The Riesz potential $u=I_{\alpha} * g\left(0<\alpha<n, I_{\alpha}(x)=|x|^{\alpha-n}\right)$ with

$$
g \in L^{n / \alpha}\left(\mathbb{R}^{n}\right) \quad \text { and } \quad \operatorname{supp} g \subset \Omega
$$

satisfies

$$
\exp \left(a|u|^{n /(n-\alpha)}\right) \in L_{\mathrm{loc}}^{1}\left(\mathbb{R}^{n}\right)
$$

for any $a \in(0, \infty)$. We refer to $[\mathrm{Z}]$ for an excellent account of this result and its improvements.

In this context a question appears: What can be said about the integrability of the Riesz potential if we replace condition (1.1) by one a little stronger, namely, by the assumption

$$
(g)_{n / \alpha, \lambda}:=\int_{\Omega}|g(x)|^{n / \alpha} \log ^{\lambda}(e+|g(x)|) \mathrm{d} x<\infty \quad \text { and } \quad \operatorname{supp} g \subset \Omega .
$$


It will be shown (cf. Remark 3.11(iii)) that this small change of integrability of $g$ causes a substantial improvement of the integrability of the Riesz potential $u=I_{\alpha} * g$ provided that $\lambda \geq n / \alpha-1$. More precisely, if $\operatorname{supp} g \subset \Omega,(g)_{n / \alpha, \lambda}<$ $\infty$ and $\lambda \geq n / \alpha-1$, then

$$
\exp \left(a_{2} \exp \left[a_{1}|u|^{n /(n-\alpha)}\right]\right) \in L_{\mathrm{loc}}^{1}\left(\mathbb{R}^{n}\right)
$$

for any $a_{1}, a_{2} \in(0, \infty)$ (that is, the Riesz potential is doubly exponentially integrable).

This is a consequence of our more general results on convolution operators in so-called generalized Lorentz-Zygmund spaces. These results extend those of Brézis-Wainger [BW] on convolution of functions in Lorentz spaces which lead to single exponential integrability.

In the above-mentioned paper, Brézis and Wainger used the following, single limiting case of Hardy's inequality

$$
\left(\int_{0}^{1}\left[(1+|\log t|)^{-1} \int_{t}^{1} g(\tau) \mathrm{d} \tau\right]^{r} \frac{\mathrm{d} t}{t}\right)^{1 / r} \leq \frac{r}{r-1}\left(\int_{0}^{1}[\operatorname{tg}(t)]^{r} \frac{\mathrm{d} t}{t}\right)^{1 / r}
$$

where $r>1$ and $g$ is a non-negative measurable function on $(0,1)$. It is possible to show that replacement of (1.2) by a more general, single limiting form of Hardy's inequality

$$
\begin{aligned}
& \left(\int_{0}^{1}\left[(1+|\log t|)^{\gamma / q} \int_{t}^{1} g(\tau) \mathrm{d} \tau\right]^{q} \frac{\mathrm{d} t}{t}\right)^{1 / q} \\
\leq & C\left(\int_{0}^{1}\left[(1+|\log t|)^{\delta / r} \operatorname{tg}(t)\right]^{r} \frac{\mathrm{d} t}{t}\right)^{1 / r}
\end{aligned}
$$

(here $q, r \in(1, \infty)$ and $\gamma, \delta \in \mathbb{R}$ are appropriate numbers) yields an extension of the Brézis-Wainger result to the scale of Lorentz-Zygmund spaces $L^{p, q}[\log L]^{\lambda}$ introduced in $[\mathrm{BR}]$ and gives single exponential integrability of the corresponding convolution operators (cf. Remark 3.11(iv)).

In our paper it is shown that the situation is qualitatively different if we use the double limiting case of Hardy's inequality

$$
\begin{aligned}
& \left(\int_{0}^{1}\left[\log \gamma / q(e+|\log t|) \int_{t}^{1} g(\tau) \mathrm{d} \tau\right]^{q} \frac{\mathrm{d} t}{t(e+|\log t|)}\right)^{1 / q} \\
\leq & C\left(\int_{0}^{1}\left[\log ^{\delta / r}(e+|\log t|) t(e+|\log t|) g(t)\right]^{r} \frac{\mathrm{d} t}{t(e+|\log t|)}\right)^{1 / r} .
\end{aligned}
$$


This leads to investigating convolution operators in generalized LorentzZygmund spaces $L^{p, q}[\log L]^{\lambda}[\log \log L]^{\varepsilon}$ (with the limiting exponent $\lambda$ ) and, at the end, to double exponential integrability of convolution operators. More precisely, for the particular case of the Riesz potential $I_{\alpha} * g$, we give necessary and sufficient conditions for the potential to induce a bounded linear map from an intersection of a generalized Lorentz-Zygmund space with an $L^{1}$-space into another generalized Lorentz-Zygmund space (cf. Theorem 2.2 and Remark 3.11(ii)), while for more general convolution operators the corresponding conditions are sufficient for analogous results to hold (cf. Theorem 2.1 and Remark 3.11(ii)). From this we obtain the corollary that if $g$ belongs to an intersection of an appropriate generalized Lorentz-Zygmund space with an $L^{1}$-space, then the Riesz potential $u=I_{\alpha} * g$ satisfies

$$
\exp \left(a_{2} \exp \left[a_{1}|u|^{\eta}\right]\right) \in L_{\text {loc }}^{1}\left(\mathbb{R}^{n}\right)
$$

for some $\eta \in(0, \infty)$ and any $a_{1}, a_{2} \in(0, \infty)$. Similar results can be obtained for more general convolutions.

The paper is organized as follows: Section 2 contains basic definitions and main results. All proofs and preliminary lemmas, including some remarks, are presented in Section 3.

We conclude this section with the following:

Notation. By $|Q|_{n}$ we mean the $n$-dimensional Lebesgue measure of the set $Q \subset \mathbb{R}^{n}$. If $p \in[1, \infty]$, then the conjugate number $p^{\prime}$ is defined by $1 / p+1 / p^{\prime}=1$.

Further notation used in Section 3 is given at the beginning of that section.

2. Main results. We begin with the definition of the generalized LorentzZygmund spaces $L^{p, q}[\log L]^{\lambda}[\log \log L]^{\varepsilon}$.

If $f$ is a measurable function defined on $\mathbb{R}^{n}$, the non-increasing rearrangement $f^{*}$ of $f$ is given by

$$
f^{*}(t)=\inf \left\{\tau>0 ;\left|\left\{x \in \mathbb{R}^{n} ;|f(x)|>\tau\right\}\right|_{n} \leq t\right\} .
$$

For $p, q \in(0, \infty]$ and $\lambda, \varepsilon \in \mathbb{R}$, the generalized Lorentz-Zygmund space $L^{p, q}[\log L]^{\lambda}[\log \log L]^{\varepsilon}$ consists of all functions $f$ satisfying $\|f\|_{p, q, \lambda, \varepsilon}<\infty$, where

$$
\|f\|_{p, q, \lambda, \varepsilon}=\left\{\begin{array}{l}
\left(\int_{0}^{\infty}\left[t^{1 / p}(e+|\log t|)^{\lambda} \log ^{\varepsilon}(e+|\log t|) f^{*}(t)\right]^{q} \frac{\mathrm{d} t}{t}\right)^{1 / q} \\
\text { for } q<\infty \\
\sup _{t>0} t^{1 / p}(e+|\log t|)^{\lambda} \log ^{\varepsilon}(e+|\log t|) f^{*}(t) \\
\text { for } q=\infty
\end{array}\right.
$$


Exactly as in the case of Lorentz spaces, for some parameters $p, q, \lambda, \varepsilon$, the functional (2.1) is only a quasi-norm. But it is possible to show, using a convenient type of the Hardy inequality, that for $p \in(1, \infty], q \in[1, \infty]$ and $\lambda$, $\varepsilon \in \mathbb{R}$, the functional

$$
f \longmapsto\|f \mid\|_{p, q, \lambda, \varepsilon},
$$

with $\||\cdot|\|_{p, q, \lambda, \varepsilon}$ defined as $(2.1)$, replacing only $f^{*}(t)$ by

$$
f^{* *}(t):=\frac{1}{t} \int_{0}^{t} f^{*}(s) \mathrm{d} s,
$$

defines a norm on $L^{p, q}[\log L]^{\lambda}[\log \log L]^{\varepsilon}$ which is equivalent to a quasi-norm (2.1). In particular, the space $L^{p, q}[\log L]^{\lambda}[\log \log L]^{\varepsilon}$ is (equivalent to) a Banach function space. Let us mention that for $\lambda=\varepsilon=0$ this space coincides with the classical Lorentz space $L^{p, q}$, with norm $\|\cdot\|_{p, q}$, while for $\varepsilon=0$ it is (equivalent to) the Lorentz-Zygmund space $L^{p, q}[\log L]^{\lambda}$, endowed with the norm $\|\cdot\|_{p, q, \lambda}$, which is introduced in [BR]. Consequently, for $\varepsilon=0$ and $p=q$, we obtain the Zygmund space $L^{p}[\log L]^{\lambda}$ (cf. [BR]), and if in addition $\lambda=0$, we have the classical Lebesgue space $L^{p}$ with the norm $\|\cdot\|_{p}$, which is a Banach function space also for $p=1$. For more details we refer the reader to [L], [S, Theorem 4], $[\mathrm{BR}]$ and $[\mathrm{BS}]$.

Throughout the paper the symbol $I_{\alpha}, \alpha \in(0, n)$, is used to denote the kernel of the Riesz potential, i.e., $I_{\alpha}(x)=|x|^{\alpha-n}, x \in \mathbb{R}^{n}$.

For deriving the double exponential integrability of convolution operators we use the following basic results which characterize boundedness of convolution operators in generalized Lorentz-Zygmund spaces.

Theorem 2.1. Let $p, q \in(1, \infty), \gamma, \delta \in \mathbb{R}$. Let $q_{1}, q_{2} \in(1, \infty]$ be such that $0<1 / q_{1}+1 / q_{2}<1$ and set $1 / r=1 / q_{1}+1 / q_{2}$. Assume

$$
f \in L^{p, q_{1}}, \quad g \in L^{p^{\prime}, q_{2}}[\log L]^{1 / r^{\prime}}[\log \log L]^{\delta / r} \cap L^{1}\left(\mathbb{R}^{n}\right), \quad u=f * g,
$$

and suppose that

$$
\begin{cases}\text { either } & r \leq q, \quad \gamma<-1, \quad \frac{\gamma+1}{q}-\frac{\delta+1}{r}+1 \leq 0, \\ \text { or } & r>q, \quad \gamma<-1, \quad \frac{\gamma+1}{q}-\frac{\delta+1}{r}+1<0 .\end{cases}
$$

Let $K$ be an arbitrary number from $(0, \infty)$. Then,

$$
\begin{aligned}
& \left(\int_{0}^{K}\left[\log ^{\gamma / q}(e+|\log t|) \cdot u^{*}(t)\right]^{q} \frac{\mathrm{d} t}{t(e+|\log t|)}\right)^{1 / q} \\
& \leq C\|f\|_{p, q_{1}} \cdot\left(\|g\|_{p^{\prime}, q_{2}, 1 / r^{\prime}, \delta / r}+\|g\|_{1}\right),
\end{aligned}
$$

where the constant $C$ depends only on $p, q, q_{1}, q_{2}, \gamma, \delta$ and $K$. 
In the special case when the function $u$ is a Riesz potential of the function $g$, the condition (2.3) is also necessary. We have the following

Theorem 2.2. Let $q, r \in(1, \infty), \alpha \in(0, n), \gamma, \delta \in \mathbb{R}$. Suppose that

$$
g \in L^{n / \alpha, r}[\log L]^{1 / r^{\prime}}[\log \log L]^{\delta / r} \cap L^{1}\left(\mathbb{R}^{n}\right) \quad \text { and } \quad u=I_{\alpha} * g
$$

If $K$ is an arbitrary number from $(0, \infty)$, then the inequality

$$
\begin{aligned}
& \left(\int_{0}^{K}\left[\log \gamma / q(e+|\log t|) \cdot u^{*}(t)\right]^{q} \frac{\mathrm{d} t}{t(e+|\log t|)}\right)^{1 / q} \\
\leq & C \cdot\left(\|g\|_{n / \alpha, r, 1 / r^{\prime}, \delta / r}+\|g\|_{1}\right)
\end{aligned}
$$

holds with a constant $C$ which depends only on $q, r, \alpha, \gamma, \delta, n$ and $K$, if and only if condition (2.3) is satisfied.

As a consequence of Theorem 2.1 and the sufficiency part of Theorem 2.2 we have the following two corollaries.

Corollary 2.3. Let the assumptions of Theorem 2.1 be satisfied. Then

(i) For any $a_{1}, a_{2} \in(0, \infty)$,

$$
\exp \left(a_{2} \exp \left[a_{1}|u|^{-q /(\gamma+1)}\right]\right) \in L_{\mathrm{loc}}^{1}\left(\mathbb{R}^{n}\right)
$$

(ii) Given any $a \in(0, \infty)$ and any measurable set $Q \subset \mathbb{R}^{n},|Q|_{n}<\infty$, there exist positive numbers $A=A\left(p, q, q_{1}, q_{2}, \gamma, \delta\right), M=M\left(a,|Q|_{n}\right)$ such that

$$
\int_{Q} \exp \left(a \exp \left[A|u(x)|^{-q /(\gamma+1)}\right]\right) \mathrm{d} x \leq M
$$

for all functions $f$ and $g$ with

$$
\|f\|_{p, q_{1}} \leq 1 \quad \text { and } \quad\|g\|_{p^{\prime}, q_{2}, 1 / r^{\prime}, \delta / r}+\|g\|_{1} \leq 1
$$

Corollary 2.4. Let all the assumptions of Theorem 2.2 and the condition (2.3) be satisfied. Then

(i) The relation $(2.6)$ holds for any $a_{1}, a_{2} \in(0, \infty)$.

(ii) Given any $a \in(0, \infty)$ and any measurable set $Q \subset \mathbb{R}^{n},|Q|_{n}<\infty$, there exist positive numbers $A=A(n, q, r, \alpha, \gamma, \delta), M=M\left(a,|Q|_{n}\right)$ such that (2.7) holds for all $g$ with $\|g\|_{n / \alpha, r, 1 / r^{\prime}, \delta / r}+\|g\|_{1} \leq 1$. 
The corollaries show that the condition (2.3) is sufficient for the double exponential integrability (2.7). In the case of the Riesz potential the following theorem goes some way towards proving necessity.

Theorem 2.5. Let $q, r \in(1, \infty), \alpha \in(0, n), \gamma, \delta \in \mathbb{R}$. Suppose that

$$
\begin{cases}\text { either } & \gamma<-1 \quad \text { and } \quad \frac{\gamma+1}{q}-\frac{\delta+1}{r}+1>0, \\ \text { or } & \gamma>-1 .\end{cases}
$$

Then for any non-empty open set $Q \subset \mathbb{R}^{n}$ and for any $a_{1}, a_{2} \in(0, \infty)$,

$\sup \left\{\int_{Q} \exp \left(a_{2} \exp \left[a_{1}\left|\left(I_{\alpha} * g\right)(x)\right|^{-q /(\gamma+1)}\right]\right) \mathrm{d} x ;\|g\|_{n / \alpha, r, 1 / r^{\prime}, \delta / r}+\|g\|_{1} \leq 1\right\}=\infty$.

3. Proofs and remarks. Throughout this section we shall use the following.

Notation 3.1. Let $0<K \leq \infty$. By $\mathfrak{M}^{+}(0, K)$, we denote the set of all non-negative measurable functions on $(0, K)$. If $f \in \mathfrak{M}^{+}(0, K)$, the symbol $f \downarrow$ means that the function $f$ is non-increasing on $(0, K)$. For $\rho \in(0, \infty)$ and $x \in \mathbb{R}^{n}$ let $B(x, \rho)$ denote the ball of radius $\rho$ and centre $x$; the symbol $\omega_{n}$ stands for the volume of a unit ball in $\mathbb{R}^{n}$. The characteristic function of a set $Q$ is denoted by $\chi_{Q}$. As usual, the symbols $C, C_{1}, C_{2}, \ldots$ signify positive constants independent of appropriate quantities. For non-negative expressions (i.e., functions or functionals) $A, B$, we use the symbol $A \approx B$ if $C_{1} B \leq A \leq$ $C_{2} B$ with some constants $C_{1}, C_{2} \in(0, \infty)$ independent of the variables in the expressions $A, B$. The symbols $x, y, z$ are strictly used for points of $\mathbb{R}^{n}$ while $s$, $t, \sigma, \tau$ are reserved for elements of $(0, \infty)$. We shall use the following convention:

$$
\infty^{0}=1, \quad \frac{1}{\infty}=0, \quad \text { and } \quad \frac{0}{0}=0 .
$$

To prove Theorem 2.1 we need the following lemmas.

Lemma 3.2. Let $0<K<\infty, q, r \in(1, \infty), \gamma, \delta \in \mathbb{R}$. Then there exists a constant $C=C(r, q, \gamma, \delta, K) \in(0, \infty)$ such that the inequality

$$
\begin{aligned}
& \left(\int_{0}^{K}\left[\log \gamma / q(e+|\log t|) \int_{t}^{K} h(s) \mathrm{d} s\right]^{q} \frac{\mathrm{d} t}{t(e+|\log t|)}\right)^{1 / q} \\
\leq & C\left(\int_{0}^{K}\left[t(e+|\log t|) \log ^{\delta / r}(e+|\log t|) h(t)\right]^{r} \frac{\mathrm{d} t}{t(e+|\log t|)}\right)^{1 / r}
\end{aligned}
$$


holds for all functions $h \in \mathfrak{M}^{+}(0, K)$ if and only if

$$
\begin{cases}\text { either } & r \leq q, \quad \gamma<-1, \quad \frac{\gamma+1}{q}-\frac{\delta+1}{r}+1 \leq 0 \\ \text { or } & r>q, \quad \gamma<-1, \quad \frac{\gamma+1}{q}-\frac{\delta+1}{r}+1<0\end{cases}
$$

is satisfied.

Proof. The inequality (3.1) can be rewritten to the form

$$
\left(\int_{0}^{K}\left(\int_{t}^{K} h(s) \mathrm{d} s\right)^{q} w(t) \mathrm{d} t\right)^{1 / q} \leq C\left(\int_{0}^{K} h^{r}(t) v(t) \mathrm{d} t\right)^{1 / r}
$$

where

$$
\begin{aligned}
& w(t)=t^{-1}(e+|\log t|)^{-1} \cdot \log ^{\gamma}(e+|\log t|), \\
& v(t)=t^{r-1}(e+|\log t|)^{r-1} \cdot \log ^{\delta}(e+|\log t|),
\end{aligned}
$$

and the result follows, e.g., from [OK, Theorems 6.2 and 6.3].

Lemma 3.3. Suppose that $p \in(1, \infty), q \in[1, \infty], \lambda, \varepsilon \in \mathbb{R}$ and that $g \in L^{p, q}[\log L]^{\lambda}[\log \log L]^{\varepsilon}$. Then there exists a positive constant $C=C(p, q, \lambda, \varepsilon)$ such that for all $s>0$,

$$
g^{*}(s) \leq g^{* *}(s) \leq C s^{-1 / p}(e+|\log s|)^{-\lambda} \log ^{-\varepsilon}(e+|\log s|)\|g\|_{p, q, \lambda, \varepsilon} .
$$

Proof. The first inequality is obvious. To prove the second one, we use the fact that the quasinorm (2.1) and the norm (2.2) are (under our assumptions) equivalent. If $q=\infty$, the desired inequality follows immediately from the definition of the norm (2.2). If $q \in[1, \infty)$, then for all $s>0$,

$$
\begin{aligned}
\||| g \mid\|_{p, q, \lambda, \varepsilon} & \geq g^{* *}(s)\left(\int_{0}^{s}\left[t^{1 / p}(e+|\log t|)^{\lambda} \log ^{\varepsilon}(e+|\log t|)\right]^{q} \frac{\mathrm{d} t}{t}\right)^{1 / q} \\
& \approx g^{* *}(s) s^{1 / p}(e+|\log s|)^{\lambda} \log ^{\varepsilon}(e+|\log s|)
\end{aligned}
$$

and the result follows.

Now we are ready to prove Theorem 2.1. 
Proof of Theorem 2.1. Since $u=f * g$, O'Neil's Lemma (see [O, Lemma 1.5] or [Z, Lemma 1.8.8]) implies that

$$
u^{*}(t) \leq u^{* *}(t) \leq t f^{* *}(t) g^{* *}(t)+\int_{t}^{\infty} f^{*}(s) g^{*}(s) \mathrm{d} s .
$$

By Lemma 3.3 we have for all $s>0$,

$$
\begin{aligned}
& \text { (3.4) } \quad f^{*}(s) \leq f^{* *}(s) \leq C_{1} s^{-1 / p}\|f\|_{p, q_{1}}, \\
& g^{*}(s) \leq g^{* *}(s) \leq C_{2} s^{-1 / p^{\prime}}(e+|\log s|)^{-1 / r^{\prime}} \log ^{-\delta / r}(e+|\log s|) \cdot\|g\|_{p^{\prime}, q_{2}, 1 / r^{\prime}, \delta / r} .
\end{aligned}
$$

Together with (3.3) these estimates yield for $t \in(0, K)$,

$$
\begin{gathered}
u^{*}(t) \leq C_{1} C_{2}\|f\|_{p, q_{1}}\|g\|_{p^{\prime}, q_{2}, 1 / r^{\prime}, \delta / r}(e+|\log t|)^{-1 / r^{\prime}} \log ^{-\delta / r}(e+|\log t|) \\
+\int_{K}^{\infty} f^{*}(s) g^{*}(s) \mathrm{d} s+\int_{t}^{K} f^{*}(s) g^{*}(s) \mathrm{d} s .
\end{gathered}
$$

Using (3.4) and the obvious inequality

$$
g^{*}(s) \leq g^{* *}(s) \leq s^{-1}\|g\|_{1}, \quad s>0,
$$

we obtain

$$
\int_{K}^{\infty} f^{*}(s) g^{*}(s) \mathrm{d} s \leq C_{1}\|f\|_{p, q_{1}} \cdot\|g\|_{1} \int_{K}^{\infty} s^{-(1+1 / p)} \mathrm{d} s=C_{3}\|f\|_{p, q_{1}} \cdot\|g\|_{1} .
$$

Defining on $\mathfrak{M}^{+}(0, K)$ the functional

$$
J_{q}(\cdot)=\left(\int_{0}^{K}(\cdot)^{q} \frac{\mathrm{d} t}{t(e+|\log t|)}\right)^{1 / q} \quad \text { for } q \in(1, \infty),
$$

the estimates (3.5), (3.6) and the sublinearity of the functional $J_{q}$ imply

$$
\begin{aligned}
& J_{q}\left(\log ^{\gamma / q}(e+|\log t|) u^{*}(t)\right) \\
\leq & C_{1} C_{2}\|f\|_{p, q_{1}} \cdot\|g\|_{p^{\prime}, q_{2}, 1 / r^{\prime}, \delta / r} \cdot \mathcal{J}_{1}+C_{3}\|f\|_{p, q_{1}} \cdot\|g\|_{1} \cdot \mathcal{J}_{2}+\mathcal{J}_{3},
\end{aligned}
$$

where

$$
\begin{aligned}
& \mathcal{J}_{1}=J_{q}\left((e+|\log t|)^{-1 / r^{\prime}} \cdot \log ^{\gamma / q-\delta / r}(e+|\log t|)\right), \\
& \mathcal{J}_{2}=J_{q}\left(\log ^{\gamma / q}(e+|\log t|)\right), \\
& \mathcal{J}_{3}=J_{q}\left(\log ^{\gamma / q}(e+|\log t|) \cdot \int_{t}^{K} f^{*}(s) g^{*}(s) \mathrm{d} s\right) .
\end{aligned}
$$


One can easily verify that

$$
\mathcal{J}_{1}<\infty, \quad \mathcal{J}_{2}<\infty
$$

Moreover, using Lemma 3.2 and the Hölder inequality (with respect to the measure $\mathrm{d} t / t)$, we obtain

$$
\begin{aligned}
\mathcal{J}_{3} \leq C(K) J_{r}\left(t(e+|\log t|) \log ^{\delta / r}(e+|\log t|) f^{*}(t) g^{*}(t)\right) \\
=C(K)\left(\int_{0}^{K}\left[t(e+|\log t|)^{1 / r^{\prime}} \log ^{\delta / r}(e+|\log t|) f^{*}(t) g^{*}(t)\right]^{r} \frac{\mathrm{d} t}{t}\right)^{1 / r} \\
\leq C(K)\left(\int_{0}^{K}\left[t^{1 / p} f^{*}(t)\right]^{q_{1}} \frac{\mathrm{d} t}{t}\right)^{1 / q_{1}} \\
\\
\qquad\left(\int_{0}^{K}\left[t^{1 / p^{\prime}}(e+|\log t|)^{1 / r^{\prime}} \log ^{\delta / r}(e+|\log t|) g^{*}(t)\right]^{q_{2}} \frac{\mathrm{d} t}{t}\right)^{1 / q_{2}} \\
\leq C(K)\|f\|_{p, q_{1}} \cdot\|g\|_{p^{\prime}, q_{2}, 1 / r^{\prime}, \delta / r} .
\end{aligned}
$$

The result follows from inequalities (3.7)-(3.9).

Proof of the sufficiency part of Theorem 2.2. For $I_{\alpha}(x)=|x|^{\alpha-n}$, $x \in \mathbb{R}^{n}$, one can easily compute that

$$
\left(I_{\alpha}\right)^{*}(t)=\left(\frac{t}{\omega_{n}}\right)^{(\alpha-n) / n}, \quad t>0
$$

(recall that $\omega_{n}$ is the volume of the unit ball in $\mathbb{R}^{n}$ ). This implies that $I_{\alpha} \in$ $L^{n /(n-\alpha), \infty}$ (cf. [Z, the text preceding Theorem 2.10.2]), and the result easily follows from Theorem 2.1 on putting $p=n /(n-\alpha)$ and $q_{1}=\infty$.

The proof of the necessity part of Theorem 2.2 is based on a method due to Sawyer [S]. Preliminary results are formulated in the following three lemmas.

Lemma 3.4 ([S, (1.20)]). Let $\alpha \in(0, n)$. For a measurable function $g: \mathbb{R}^{n} \rightarrow[-\infty, \infty]$ let us write $\tilde{g}(y)=g^{*}\left(\omega_{n}|y|^{n}\right), y \in \mathbb{R}^{n}$. Then for all $t>0$,

$$
\begin{aligned}
\frac{\alpha}{n} \omega_{n}{ }^{\alpha / n-1}\left(I_{\alpha} * g\right)^{*}(t) & \leq t^{\alpha / n-1} \int_{0}^{t} g^{*}(s) \mathrm{d} s+\int_{t}^{\infty} s^{\alpha / n-1} g^{*}(s) \mathrm{d} s \\
& \leq 2^{n-\alpha} \omega_{n}^{\alpha / n-1}\left(I_{\alpha} * \tilde{g}\right)^{*}(t)
\end{aligned}
$$


Proof. The first inequality in (3.11) follows from (3.3), (3.10) and its immediate consequence $\left(I_{\alpha}\right)^{* *}(t)=(n / \alpha)\left(t / \omega_{n}\right)^{(\alpha-n) / n}, t>0$. Now we prove the second inequality in (3.11). Let us estimate

$$
\begin{aligned}
\left(I_{\alpha} * \tilde{g}\right)(x) & =\int_{\mathbb{R}^{n}} \frac{\tilde{g}(y)}{|x-y|^{n-\alpha}} \mathrm{d} y \\
& \geq \frac{1}{2^{n-\alpha}}\left[\int_{|y|<|x|} \frac{\tilde{g}(y)}{|x|^{n-\alpha}} \mathrm{d} y+\int_{|y| \geq|x|} \frac{\tilde{g}(y)}{|y|^{n-\alpha}} \mathrm{d} y\right], \quad x \in \mathbb{R}^{n} .
\end{aligned}
$$

Using spherical coordinates we obtain

$$
\begin{aligned}
& \int_{|y|<|x|} \frac{\tilde{g}(y)}{|x|^{n-\alpha}} \mathrm{d} y \\
= & \frac{1}{|x|^{n-\alpha}} \int_{|y|<|x|} g^{*}\left(\omega_{n}|y|^{n}\right) \mathrm{d} y \\
= & |x|^{\alpha-n} \int_{0}^{|x|} \int_{\{|y|=r\}} g^{*}\left(\omega_{n} r^{n}\right) \mathrm{d} \vartheta \mathrm{d} r \\
= & |x|^{\alpha-n} \int_{0}^{|x|} g^{*}\left(\omega_{n} r^{n}\right) \omega_{n} n r^{n-1} \mathrm{~d} r=|x|^{\alpha-n} \int_{0}^{\omega_{n}|x|^{n}} g^{*}(s) \mathrm{d} s \\
= & \omega_{n}{ }^{1-\alpha / n}\left(\omega_{n}|x|^{n}\right)^{\alpha / n-1} \int_{0}^{\omega_{n}|x|^{n}} g^{*}(s) \mathrm{d} s .
\end{aligned}
$$

Similarly we obtain,

$$
\int_{|y| \geq|x|} \frac{\tilde{g}(y)}{|y|^{n-\alpha}} \mathrm{d} y=\omega_{n}^{1-\alpha / n} \int_{\omega_{n}|x|^{n}}^{\infty} s^{\alpha / n-1} g^{*}(s) \mathrm{d} s .
$$

From (3.12), (3.13) and (3.14) it follows

$$
\left(I_{\alpha} * \tilde{g}\right)(x) \geq 2^{\alpha-n} \omega_{n}{ }^{1-\alpha / n} \cdot G\left(\omega_{n}|x|^{n}\right), \quad x \in \mathbb{R}^{n},
$$

where

$$
G(t)=t^{\alpha / n-1} \int_{0}^{t} g^{*}(s) \mathrm{d} s+\int_{t}^{\infty} s^{\alpha / n-1} g^{*}(s) \mathrm{d} s, \quad t>0
$$

Observing

$$
\left|\left\{y \in \mathbb{R}^{n} ; G\left(\omega_{n}|y|^{n}\right)>\tau\right\}\right|_{n}=|\{t>0 ; G(t)>\tau\}|_{1}, \quad \tau>0,
$$

we see that the functions $y \mapsto G\left(\omega_{n}|y|^{n}\right)$ and $t \mapsto G(t)$ have the same nonincreasing rearrangement. Moreover, $G$ is non-increasing on $(0, \infty)$. Thus, by (3.15),

$$
\left(I_{\alpha} * \tilde{g}\right)^{*}(t) \geq 2^{\alpha-n} \omega_{n}^{1-\alpha / n} \cdot G^{*}(t)=2^{\alpha-n} \omega_{n}^{1-\alpha / n} \cdot G(t), \quad t>0,
$$

which is the second inequality in (3.11). 
Lemma 3.5. Let $0<K \leq \infty, 1<p<\infty$, and let $v$ be a function such that $v(t)>0$ for a.e. $t \in(0, K)$ and

$$
\int_{0}^{t} v(\tau) \mathrm{d} \tau<\infty, \quad t \in(0, K) .
$$

Then for any function $g \in \mathfrak{M}^{+}(0, K)$, we have

$$
\sup _{f \in \mathfrak{M}^{+}(0, K), f \downarrow} \frac{\int_{0}^{K} f(t) g(t) \mathrm{d} t}{\left(\int_{0}^{K} f^{p}(t) v(t) \mathrm{d} t\right)^{1 / p}} \approx\left(\int_{0}^{K}\left(\int_{t}^{K} \frac{g(s)}{\int_{0}^{s} v} \mathrm{~d} s\right)^{p^{\prime}} v(t) \mathrm{d} t\right)^{1 / p^{\prime}} .
$$

For the proof see [S, proof of Theorem 1].

Lemma 3.6. Let $0<K \leq \infty$, let $w$, $v$ be functions positive a.e. on $(0, K)$ and let $v$ satisfy condition (3.16). Suppose that

$$
T, T^{\prime}: \mathfrak{M}^{+}(0, K) \longrightarrow \mathfrak{M}^{+}(0, K)
$$

are two operators satisfying the condition

$$
\int_{0}^{K} T f(t) \cdot g(t) \mathrm{d} t=\int_{0}^{K} f(t) \cdot T^{\prime} g(t) \mathrm{d} t
$$

for all functions $f, g \in \mathfrak{M}^{+}(0, K), f \downarrow$. Let $q, r \in(1, \infty)$. Then the inequality

$$
\left(\int_{0}^{K}(T f)^{q}(t) w(t) \mathrm{d} t\right)^{1 / q} \leq C\left(\int_{0}^{K} f^{r}(t) v(t) \mathrm{d} t\right)^{1 / r}
$$

holds for all $f \in \mathfrak{M}^{+}(0, K), f \downarrow$, if and only if the inequality

$$
\left(\int_{0}^{K}\left(\int_{t}^{K} \frac{T^{\prime} g(s)}{\int_{0}^{s} v} \mathrm{~d} s\right)^{r^{\prime}} v(t) \mathrm{d} t\right)^{1 / r^{\prime}} \leq C\left(\int_{0}^{K} g^{q^{\prime}}(t) w^{1-q^{\prime}}(t) \mathrm{d} t\right)^{1 / q^{\prime}}
$$

holds for all $g \in \mathfrak{M}^{+}(0, K)$ (the constant $C$ is the same in both inequalities and does not depend on the functions $f, g)$.

Proof. The assertion is an easy consequence of Lemma 3.5, the Hölder inequality and (3.17); we leave it to the reader. 
Remark 3.7. In the following we use the obvious fact that any function $\varphi \in \mathfrak{M}^{+}(0, K)(0<K \leq \infty)$ can be associated with the function $g: \mathbb{R}^{n} \rightarrow[0, \infty]$ which satisfies

$$
g^{*}(t)=\varphi(t) \quad \text { for a.a. } t \in(0, K)
$$

(putting $g(x)=\varphi\left(\omega_{n}|x|^{n}\right)$ for $|x|<K$ and $g(x)=0$ otherwise).

Proof of the necessity part of Theorem 2.2. Take

$$
g: \mathbb{R}^{n} \rightarrow[0, \infty] \quad \text { with }|\operatorname{supp} g|_{n} \leq K,\|g\|_{n / \alpha, r, 1 / r^{\prime}, \delta / r}<\infty .
$$

Then there exists $C_{1}=C_{1}(n, r, \alpha, \delta, K)>0$ such that

$$
\|g\|_{1} \leq C_{1}\|g\|_{n / \alpha, r, 1 / r^{\prime}, \delta / r}
$$

Putting

$$
\tilde{g}(y)=g^{*}\left(\omega_{n}|y|^{n}\right), \quad y \in \mathbb{R}^{n},
$$

and observing that $(\tilde{g})^{*}=g^{*}$, we have

$$
\|\tilde{g}\|_{n / \alpha, r, 1 / r^{\prime}, \delta / r}=\|g\|_{n / \alpha, r, 1 / r^{\prime}, \delta / r}, \quad\|\tilde{g}\|_{1}=\|g\|_{1} .
$$

By (2.5), (3.19) and (3.21),

$$
\left(\int_{0}^{K}\left[\log ^{\gamma / q}(e+|\log t|)\left(I_{\alpha} * \tilde{g}\right)^{*}(t)\right]^{q} \frac{\mathrm{d} t}{t(e+|\log t|)}\right)^{1 / q} \leq C\left(C_{1}+1\right)\|g\|_{n / \alpha, r, 1 / r^{\prime}, \delta / r} .
$$

According to (3.11),

$$
\left(I_{\alpha} * \tilde{g}\right)^{*}(t) \geq C_{2} \int_{t}^{K} s^{\alpha / n-1} g^{*}(s) \mathrm{d} s, \quad t \in(0, K),
$$

and consequently,

$$
\begin{aligned}
& \left(\int_{0}^{K}\left[\log \gamma / q(e+|\log t|) \int_{t}^{K} s^{\alpha / n-1} g^{*}(s) \mathrm{d} s\right]^{q} \frac{\mathrm{d} t}{t(e+|\log t|)}\right)^{1 / q} \\
\leq & C_{3}\left(\int_{0}^{K}\left[t^{\alpha / n}(e+|\log t|)^{1 / r^{\prime}} \log ^{\delta / r}(e+|\log t|) g^{*}(t)\right]^{r} \frac{\mathrm{d} t}{t}\right)^{1 / r} .
\end{aligned}
$$

This easily implies (cf. Remark 3.7) that the inequality

$$
\left(\int_{0}^{K}(T f)^{q}(t) w(t) \mathrm{d} t\right)^{1 / q} \leq C\left(\int_{0}^{K} f^{r}(t) v(t) \mathrm{d} t\right)^{1 / r}
$$


(with $C=C_{3}$ ) holds for all $f \in \mathfrak{M}^{+}(0, K), f \downarrow$, where

$$
\begin{gathered}
T f(t)=\int_{t}^{K} s^{\alpha / n-1} f(s) \mathrm{d} s, \\
w(t)=t^{-1}(e+|\log t|)^{-1} \log ^{\gamma}(e+|\log t|), \\
v(t)=t^{(\alpha / n) r-1}(e+|\log t|)^{r-1} \log ^{\delta}(e+|\log t|)
\end{gathered}
$$

for $t \in(0, K)$. Obviously, $v$ satisfies (3.16).

Defining the operator $T^{\prime}: \mathfrak{M}^{+}(0, K) \rightarrow \mathfrak{M}^{+}(0, K)$ by

$$
T^{\prime} g(s)=s^{\alpha / n-1} \int_{0}^{s} g(\sigma) \mathrm{d} \sigma, \quad s \in(0, K),
$$

the Fubini theorem yields (3.17). Consequently, applying Lemma 3.6, we obtain that the inequality (3.18) is fulfilled for all $g \in \mathfrak{M}^{+}(0, K)$. By the monotonicity of the function $s \mapsto \int_{0}^{s} g(\sigma) \mathrm{d} \sigma$ we have

$$
\int_{t}^{K} \frac{T^{\prime} g(s)}{\int_{0}^{s} v} \mathrm{~d} s \geq G^{\prime} g(t) \int_{t}^{K} s^{\alpha / n-1}\left(\int_{0}^{s} v(\sigma) \mathrm{d} \sigma\right)^{-1} \mathrm{~d} s,
$$

with $G^{\prime} g(t)=\int_{0}^{t} g(\sigma) \mathrm{d} \sigma$. Setting

$$
\bar{v}(t)=\left(\int_{t}^{K} s^{\alpha / n-1}\left(\int_{0}^{s} v(\sigma) \mathrm{d} \sigma\right)^{-1} \mathrm{~d} s\right)^{-r} v^{1-r}(t), \quad t \in(0, K),
$$

the estimate (3.22) and the inequality (3.18) imply that

$$
\left(\int_{0}^{K}\left(G^{\prime} g\right)^{r^{\prime}}(t) \bar{v}^{1-r^{\prime}}(t) \mathrm{d} t\right)^{1 / r^{\prime}} \leq C\left(\int_{0}^{K} g^{q^{\prime}}(t) w^{1-q^{\prime}}(t) \mathrm{d} t\right)^{1 / q^{\prime}}
$$

for all $g \in \mathfrak{M}^{+}(0, K)$. Hence, by the duality argument,

$$
\left(\int_{0}^{K}(G h)^{q}(t) w(t) \mathrm{d} t\right)^{1 / q} \leq C\left(\int_{0}^{K} h^{r}(t) \bar{v}(t) \mathrm{d} t\right)^{1 / r}
$$

for all $h \in \mathfrak{M}^{+}(0, K)$ with $G h(t)=\int_{t}^{K} h(s) \mathrm{d} s$. Observing that

$$
\bar{v}(t) \approx t^{r-1}(e+|\log t|)^{r-1} \log ^{\delta}(e+|\log t|)
$$

for all $t \in(0, K)$, we get the equivalence of (3.23) and (3.1) and the result follows from Lemma 3.2. 


\section{Remarks 3.8.}

(i) Applying Lemma 3.6 (cf. its application in the previous proof), it is also possible to prove the following assertion:

Let $0<K \leq \infty, q, r \in(1, \infty), \gamma, \delta \in \mathbb{R}$. Then the inequality (3.1) holds on $\mathfrak{M}^{+}(0, K)$ if and only if this inequality holds for all $h \in \mathfrak{M}^{+}(0, K), h \downarrow$.

(ii) In the following we shall employ this simple observation:

Let $\Phi:[0, \infty] \rightarrow[0, \infty]$ be a strictly monotone function. Let $Q \subset \mathbb{R}^{n}$, $|Q|_{n}<\infty$, and let $H: Q \rightarrow[0, \infty]$ be a measurable function. Then the functions

$$
x \mapsto \Phi(H(x)) \quad \text { and } \quad t \mapsto \Phi\left(H^{*}(t)\right)
$$

are equimeasurable, and consequently they have the same non-increasing rearrangement, say $F$. Hence

$$
\int_{Q} \Phi(H(x)) \mathrm{d} x=\int_{0}^{\infty} F(t) \mathrm{d} t=\int_{0}^{\infty} \Phi\left(H^{*}(t)\right) \mathrm{d} t .
$$

To prove Corollaries 2.3 and 2.4 we shall use:

Lemma 3.9. Let $0<q \leq \infty, \alpha<-1 / q$. For a measurable function $u$ on $\mathbb{R}^{n}$ put

$$
[u]_{q, \alpha}:=\left(\int_{0}^{1}\left[\log ^{\alpha}(e-\log s) u^{*}(s)\right]^{q} \frac{\mathrm{d} s}{s(e-\log s)}\right)^{1 / q}
$$

(with the usual modification for $q=\infty$ ).

(i) If $q<\infty$ and $[u]_{q, \alpha}<\infty$, then

$$
\exp \left(a_{2} \exp \left[a_{1}|u|^{-1 /(\alpha+1 / q)}\right]\right) \in L_{\mathrm{loc}}^{1}\left(\mathbb{R}^{n}\right)
$$

for any $a_{1}, a_{2} \in(0, \infty)$.

(ii) If $\mathfrak{S}$ is a non-empty set of measurable functions on $\mathbb{R}^{n}$ satisfying

$$
\sup _{u \in \mathfrak{S}}[u]_{q, \alpha}=S_{q, \alpha}<\infty
$$

given any $a \in(0, \infty), \beta \in(0,1)$ and $Q \subset \mathbb{R}^{n},|Q|_{n}<\infty$, there exists a positive constant $M=M\left(a, \beta,|Q|_{n}\right)$ such that

$$
\int_{Q} \exp \left(a \exp \left\{\beta[-(\alpha q+1)]^{1 /(\alpha q+1)}\left(\frac{|u(x)|}{S_{q, \alpha}}\right)^{-1 /(\alpha+1 / q)}\right\}\right) \mathrm{d} x \leq M
$$

for all functions $u \in \mathfrak{S}$. 
Proof. (i): Assume that $0<q<\infty, \alpha<-1 / q,[u]_{q, \alpha}<\infty$, and $a_{1}$, $a_{2} \in(0, \infty)$. Putting

$$
I(t)=\int_{0}^{t}\left[\log ^{\alpha}(e-\log s) u^{*}(s)\right]^{q} \frac{\mathrm{d} s}{s(e-\log s)},
$$

we obtain for all $t \in(0,1)$,

$$
\begin{aligned}
I(t)^{1 / q} & \geq u^{*}(t)\left(\int_{0}^{t} \log ^{\alpha q}(e-\log s) \frac{\mathrm{d} s}{s(e-\log s)}\right)^{1 / q} \\
& =u^{*}(t)[-(\alpha q+1)]^{-1 / q} \log ^{\alpha+1 / q}(e-\log t),
\end{aligned}
$$

and consequently,

$$
a_{1} u^{*}(t)^{-1 /(\alpha+1 / q)} \leq b(t) \cdot \log (e-\log t),
$$

where $b(t)=a_{1}[-(\alpha q+1) I(t)]^{-1 /(\alpha q+1)}$. Since $I(t)$ is non-decreasing on $(0,1)$, we have by (3.28) that

$$
\exp \left(a_{2} \exp \left[a_{1} u^{*}(t)^{-1 /(\alpha+1 / q)}\right]\right) \leq \exp \left[a_{2}(e-\log t)^{b\left(t_{0}\right)}\right]
$$

for any $t_{0} \in(0,1)$ and all $t \in\left(0, t_{0}\right)$. The assumption $[u]_{q, \alpha}<\infty$ implies that $I(t) \rightarrow 0$ as $t \rightarrow 0_{+}$. Thus, there is $t_{0} \in(0,1)$ satisfying $\beta:=b\left(t_{0}\right)<1$. Hence

$$
\lim _{t \rightarrow 0_{+}}(e-\log t)^{\beta} /(-\log t)=0,
$$

and so there exists $t_{1} \in\left(0, t_{0}\right)$ such that

$$
a_{2}(e-\log t)^{\beta} \leq \frac{1}{2}(-\log t)=\log t^{-1 / 2}, \quad t \in\left(0, t_{1}\right) .
$$

This and (3.29) yields

$$
\exp \left(a_{2} \exp \left[a_{1} u^{*}(t)^{-1 /(\alpha+1 / q)}\right]\right) \leq t^{-1 / 2}, \quad t \in\left(0, t_{1}\right) .
$$

Consequently, since $u^{*}$ is non-increasing,

$$
\exp \left(a_{2} \exp \left[a_{1} u^{*}(t)^{-1 /(\alpha+1 / q)}\right]\right) \leq t_{1}^{-1 / 2}, \quad t \in\left[t_{1}, \infty\right) .
$$

With any measurable set $Q \subset \mathbb{R}^{n},|Q|_{n} \in(1, \infty)$, we have (cf. Remark 3.8 (ii))

$$
\begin{aligned}
& \int_{Q} \exp \left(a_{2} \exp \left[a_{1}|u(x)|^{-1 /(\alpha+1 / q)}\right]\right) \mathrm{d} x \\
\leq & \int_{0}^{|Q|_{n}} \exp \left(a_{2} \exp \left[a_{1} u^{*}(t)^{-1 /(\alpha+1 / q)}\right]\right) \mathrm{d} t \\
\leq & \int_{0}^{t_{1}} t^{-1 / 2} \mathrm{~d} t+\int_{t_{1}}^{|Q|_{n}} t_{1}^{-1 / 2} \mathrm{~d} t=C\left(t_{1},|Q|_{n}\right)<\infty
\end{aligned}
$$


and (3.25) is verified.

(ii-1): If $0<q<\infty, \alpha<-1 / q$ and $[u]_{q, \alpha}<\infty$, then (3.27) implies

$$
\begin{aligned}
& {[u]_{\infty, \alpha+1 / q}=\sup _{t \in(0,1)} u^{*}(t) \log ^{\alpha+1 / q}(e-\log t) } \\
\leq & {[-(\alpha q+1)]^{1 / q} \cdot I(1)^{1 / q}=[-(\alpha q+1)]^{1 / q} \cdot[u]_{q, \alpha} . }
\end{aligned}
$$

This estimate indicates that it suffices to prove (3.26) for $q=\infty$ only.

(ii-2): Let $\alpha<0, \sup _{u \in \mathfrak{S}}[u]_{\infty, \alpha}=S_{\infty, \alpha}<\infty, a \in(0, \infty)$ and $\beta \in(0,1)$. Then for all $u \in \mathfrak{S}$ and $t \in(0,1)$,

$$
u^{*}(t) \log ^{\alpha}(e-\log t) \leq[u]_{\infty, \alpha} \leq S_{\infty, \alpha},
$$

and consequently

$$
\beta\left(\frac{u^{*}(t)}{S_{\infty, \alpha}}\right)^{-1 / \alpha} \leq \log (e-\log t)^{\beta}
$$

Hence,

$$
\exp \left(a \exp \left\{\beta\left(\frac{u^{*}(t)}{S_{\infty, \alpha}}\right)^{-1 / \alpha}\right\}\right) \leq \exp \left[a(e-\log t)^{\beta}\right]
$$

for all $u \in \mathfrak{S}$, and $t \in(0,1)$. Now, using estimates (3.30) (with $\left.a_{2}=a\right),(3.31)$ and the same argument as in part (i), we obtain (3.26).

Proofs of Corollaries 2.3 and 2.4. We shall prove only Corollary 2.3. The proof of Corollary 2.4 is analogous.

Taking $K=1$ in Theorem 2.1 and using the notation (3.24), the inequality (2.4) can be rewritten as

$$
[u]_{q, \gamma / q} \leq C\|f\|_{p, q_{1}}\left(\|g\|_{p^{\prime}, q_{2}, 1 / r^{\prime}, \delta / r}+\|g\|_{1}\right) .
$$

The results now follow on taking $\alpha=\gamma / q, \beta=1 / 2, \mathfrak{S}=\left\{u ;[u]_{q, \alpha} \leq C\right\}$ and $S_{q, \alpha}=C$ in Lemma 3.9 .

To prove Theorem 2.5 we need:

Lemma 3.10. Let $K \in(0, \infty), \alpha<0$ and let $\mathfrak{S} \subset \mathfrak{M}^{+}(0, K), \mathfrak{S} \neq \varnothing$. Then the following statements are equivalent.

(i) There exist $a_{1}, a_{2}, M \in(0, \infty)$ such that

$$
\int_{0}^{K} \exp \left(a_{2} \exp \left[a_{1} f(t)^{-1 / \alpha}\right]\right) \mathrm{d} t \leq M \quad \text { for all } f \in \mathfrak{S} .
$$

(ii) There exists $B \in(0, \infty)$ such that

$$
\sup _{0<t<K} f^{*}(t) \log ^{\alpha}(e+|\log t|) \leq B \quad \text { for all } f \in \mathfrak{S} \text {. }
$$


Proof. It is easy to see that (3.32) is equivalent to the inequality (cf. Remark $3.8($ ii) $)$

$$
\int_{0}^{K} \exp \left[a_{2} \exp \left(a_{1} f^{*}(t)^{-1 / \alpha}\right)\right] \mathrm{d} t \leq M \quad \text { for all } f \in \mathfrak{S} \text {. }
$$

Thus, it is sufficient to show that $(3.33) \Leftrightarrow\left(3.32^{*}\right)$.

$(3.33) \Rightarrow\left(3.32^{*}\right)$ : The proof of this implication is analogous to part (ii-2) of the proof of Lemma 3.9.

$\left(3.32^{*}\right) \Rightarrow(3.33)$ : Put

$$
\Phi_{\alpha}(\tau)=\exp \left[a_{2} \exp \left(a_{1} \tau^{-1 / \alpha}\right)\right], \quad \tau>0 .
$$

Then

$$
\Phi_{\alpha} \text { is increasing on }(0, \infty),
$$

and there exists $\tau_{0} \in[0, \infty)$ such that $\Phi_{\alpha}$ is convex on $\left(\tau_{0}, \infty\right)$ and

$$
\Phi_{\alpha}(\tau)>\tau \quad \text { for } \tau \in\left(\tau_{0}, \infty\right) .
$$

Take $f \in \mathfrak{S}$ and set

$$
\begin{aligned}
& E_{f}=\left\{t \in(0, K) ; f^{*}(t) \leq \tau_{0}\right\}, \\
& F_{f}=\left\{t \in(0, K) ; f^{*}(t)>\tau_{0}\right\} .
\end{aligned}
$$

If $E_{f} \neq \varnothing$, then

$$
\begin{aligned}
\sup _{t \in E_{f}} f^{*}(t) \log ^{\alpha}(e+|\log t|) & \leq \tau_{0} \sup _{0<t<K} \log ^{\alpha}(e+|\log t|) \\
& =C\left(\tau_{0}, \alpha, K\right)<\infty .
\end{aligned}
$$

Let $F_{f} \neq \varnothing$ (otherwise the result immediately follows from (3.38)). Since $f^{*}$ is non-increasing and right-continuous on $(0, \infty)$, there exists a number $c_{f} \in$ $(0, K]$ such that

$$
F_{f}=\left(0, c_{f}\right)
$$

We intend to use the Jensen inequality (see, e.g., $[\mathrm{R}]$ ),

$$
\Phi_{\alpha}\left(\frac{1}{t} \int_{0}^{t} f^{*}(s) \mathrm{d} s\right) \leq \frac{1}{t} \int_{0}^{t} \Phi_{\alpha}\left(f^{*}(s)\right) \mathrm{d} s, \quad t \in F_{f} .
$$


For this we need to verify that

$$
\begin{aligned}
f^{*}((0, t)) & \subset\left(\tau_{0}, \infty\right), \\
f^{*} & \in L^{1}(0, t)
\end{aligned}
$$

for any $t \in F_{f}$.

The inclusion (3.41) is a consequence of (3.39) and (3.37). For proving (3.42) we use $(3.36),(3.35)$ and $\left(3.32^{*}\right)$ to get

$$
\begin{aligned}
\int_{0}^{t} f^{*}(s) \mathrm{d} s & \leq \int_{0}^{K} f^{*}(t) \mathrm{d} t=\int_{E_{f}} f^{*}(t) \mathrm{d} t+\int_{F_{f}} f^{*}(s) \mathrm{d} s \\
& \leq \tau_{0} K+\int_{F_{f}} \Phi_{\alpha}\left(f^{*}(t)\right) \mathrm{d} t \leq \tau_{0} K+M, \quad t \in F_{f} .
\end{aligned}
$$

Now, by (3.40) and $\left(3.32^{*}\right)$, we obtain

$$
\Phi_{\alpha}\left(\frac{1}{t} \int_{0}^{t} f^{*}(s) \mathrm{d} s\right) \leq \frac{M}{t}
$$

Using the obvious inequality $f^{*}(t) \leq t^{-1} \int_{0}^{t} f^{*}(s) \mathrm{d} s, t>0,(3.34)$ and (3.43), we have

$$
\Phi_{\alpha}\left(f^{*}(t)\right) \leq \frac{M}{t} \quad \text { for all } t \in F_{f} .
$$

Without loss of generality we can assume that $M \geq 2 K$. Then, the definition of $\Phi_{\alpha}$ and (3.44) yield

$$
f^{*}(t) \leq a_{1}^{\alpha} \log ^{-\alpha}\left(a_{2}^{-1} \log \frac{M}{t}\right), \quad t \in F_{f}
$$

and consequently,

$$
\begin{aligned}
\sup _{t \in F_{f}} f^{*}(t) \log ^{\alpha}(e+|\log t|) & \leq a_{1}^{\alpha} \sup _{0<t<K}\left[\log ^{\alpha}(e+|\log t|) \cdot \log ^{-\alpha}\left(a_{2}^{-1} \log \frac{M}{t}\right)\right] \\
& =C\left(a_{1}, a_{2}, K, M, \alpha\right)<\infty,
\end{aligned}
$$

which together with (3.38) gives (3.33).

Proof of Theorem 2.5. Set $\Phi(t)=\exp \left[a_{2} \exp \left(a_{1} t^{-q /(\gamma+1)}\right)\right], t>0$. Assume that the assertion of Theorem 2.5 does not hold. Then there are numbers $a_{1}, a_{2}, M \in(0, \infty)$ and a non-empty open set $Q \subset \mathbb{R}^{n}$ satisfying

$$
M \geq \int_{Q} \Phi\left(\left|\left(I_{\alpha} * g\right)(x)\right|\right) \mathrm{d} x
$$

for all functions $g$ with

$$
\|g\|_{n / \alpha, r, 1 / r^{\prime}, \delta / r}+\|g\|_{1} \leq 1 \text {. }
$$

Obviously, there exist $x_{0} \in \mathbb{R}^{n}$ and $\rho>0$ such that the ball $B\left(x_{0}, \rho\right) \subset Q$. Put $K=\left|B\left(x_{0}, \rho\right)\right|_{n}$.

First, suppose that $x_{0}=0$. We shall distinguish two cases: 
$\overline{\text { Case (i). }}$

Let $\gamma<-1$ and $(\gamma+1) / q-(\delta+1) / r+1>0$. Then,

$\Phi$ is increasing on $(0, \infty)$.

Take $g: \mathbb{R}^{n} \rightarrow[-\infty, \infty]$ with

$$
\operatorname{supp} g \subset B(0, \rho), \quad 0<\|g\|_{n / \alpha, r, 1 / r^{\prime}, \delta / r}<\infty .
$$

Then (3.19) holds. Defining $\tilde{g}$ by (3.20), we have (3.21). Moreover, by (3.15),

$$
\left(I_{\alpha} * \tilde{g}\right)(x) \geq C_{2} F(x), \quad x \in \mathbb{R}^{n},
$$

where $F(x)=G\left(\omega_{n}|x|^{n}\right) \chi_{B(0, \rho)}(x), C_{2}=C_{2}(\alpha, n)$; recall that $\chi_{B(0, \rho)}$ stands for the characteristic function of the ball $B(0, \rho)$. Putting $\tilde{g}_{d}=d \cdot \tilde{g}$ and taking

$$
d=\left[\left(C_{1}+1\right)\|g\|_{n / \alpha, r, 1 / r^{\prime}, \delta / r}\right]^{-1},
$$

we have $\left\|\tilde{g}_{d}\right\|_{n / \alpha, r, 1 / r^{\prime}, \delta / r}+\left\|\tilde{g}_{d}\right\|_{1} \leq 1$. Since $B(0, \rho) \subset Q$, the inequalities (3.45), (3.48), the property (3.46) and the facts that $G$ is decreasing and that $\operatorname{supp} g^{*} \subset$ $[0, K]$ yield

$$
\begin{aligned}
M & \geq \int_{B(0, \rho)} \Phi\left(\left|\left(I_{\alpha} * \tilde{g}_{d}\right)(x)\right|\right) \mathrm{d} x \geq \int_{\mathbb{R}^{n}} \Phi\left(C_{2} d F(x)\right) \mathrm{d} x \\
& =\int_{0}^{\infty} \Phi\left(C_{2} d F^{*}(t)\right) \mathrm{d} t=\int_{0}^{K} \Phi\left(C_{2} d F^{*}(t)\right) \mathrm{d} t \\
& =\int_{0}^{K} \Phi\left(C_{2} d G(t)\right) \mathrm{d} t \geq \int_{0}^{K} \Phi\left(C_{2} d \int_{t}^{K} s^{\alpha / n-1} g^{*}(s) \mathrm{d} s\right) \mathrm{d} t \\
& =\int_{0}^{K} \exp \left[a_{2} \exp \left(a_{1}\left(C_{2} d \int_{t}^{K} s^{\alpha / n-1} g^{*}(s) \mathrm{d} s\right)^{-q /(\gamma+1)}\right)\right] \mathrm{d} t
\end{aligned}
$$

for all functions $g$ satisfying (3.47). Consequently, by Lemma 3.10, there is $B \in(0, \infty)$ such that

$$
\sup _{0<t<K}\left(\int_{t}^{K} s^{\alpha / n-1} g^{*}(s) \mathrm{d} s\right) \log (\gamma+1) / q(e+|\log t|) \leq B\left(C_{2} d\right)^{-1}
$$

for the same family of functions $g$. 
Choose $\gamma_{1} \in \mathbb{R}$ such that

$$
\gamma_{1}<\gamma \quad \text { and } \quad \frac{\gamma_{1}+1}{q}-\frac{\delta+1}{r}+1>0
$$

Then for an arbitrary $h \in \mathfrak{M}^{+}(0, K)$,

$$
\begin{aligned}
& \left(\int_{0}^{K}\left[\log ^{\gamma_{1} / q}(e+|\log t|) \cdot h(t)\right]^{q} \frac{\mathrm{d} t}{t(e+|\log t|)}\right)^{1 / q} \\
\leq & C_{3} \cdot \sup _{0<t<K} h(t) \log ^{(\gamma+1) / q}(e+|\log t|),
\end{aligned}
$$

where

$$
C_{3}=\left(\int_{0}^{K} \log ^{\gamma_{1}-\gamma-1}(e+|\log t|) \frac{\mathrm{d} t}{t(e+|\log t|)}\right)^{1 / q}<\infty .
$$

Hence, by (3.50), (3.52) and (3.49),

$$
\begin{aligned}
& \left(\int_{0}^{K}\left[\log ^{\gamma_{1} / q}(e+|\log t|) \cdot \int_{t}^{K} s^{\alpha / n-1} g^{*}(s) \mathrm{d} s\right]^{q} \frac{\mathrm{d} t}{t(e+|\log t|)}\right)^{1 / q} \\
\leq & C_{4}\|g\|_{n / \alpha, r, 1 / r^{\prime}, \delta / r}
\end{aligned}
$$

with $C_{4}=B C_{3} C_{2}^{-1}\left(C_{1}+1\right)$. This implies (cf. the proof of the necessity part of Theorem 2.2) that the inequality

$$
\left(\int_{0}^{K}\left(\int_{t}^{K} h(s) \mathrm{d} s\right)^{q} w(t) \mathrm{d} t\right)^{1 / q} \leq C_{4}\left(\int_{0}^{K} h^{r}(t) v(t) \mathrm{d} t\right)^{1 / r}
$$

where

$$
\begin{aligned}
& w(t)=t^{-1}(e+|\log t|)^{-1} \log ^{\gamma_{1}}(e+|\log t|), \\
& v(t)=t^{r-1}(e+|\log t|)^{r-1} \log ^{\delta}(e+|\log t|),
\end{aligned}
$$

holds for all $h \in \mathfrak{M}^{+}(0, K)$. However, by Lemma 3.2, this is impossible for (3.51) is fulfilled.

$\overline{\text { Case (ii). }}$

Let $\gamma>-1$. Then

$\Phi$ is decreasing on $(0, \infty)$. 
For $\varepsilon>0$ put $g_{\varepsilon}=\varepsilon \cdot g$ with $g$ from (3.47). Since the function

$$
G(t)=\frac{n}{\alpha} \omega_{n}{ }^{1-\alpha / n}\left[t^{\alpha / n-1} \int_{0}^{t} g^{*}(s) \mathrm{d} s+\int_{t}^{K} s^{\alpha / n-1} g^{*}(s) \mathrm{d} s\right]
$$

is decreasing on $(0, K)$, Lemma 3.4 (applied to the function $\tilde{g}_{\varepsilon}$ instead of $g$ ) yields for any $t \in(K / 2, K)$,

$$
\left(I_{\alpha} * \tilde{g}_{\varepsilon}\right)^{*}(t) \leq \varepsilon \cdot G(t) \leq \varepsilon \cdot G\left(\frac{K}{2}\right) .
$$

Taking

$$
H(x):=\left(I_{\alpha} * \tilde{g}_{\varepsilon}\right)(x) \cdot \chi_{B(0, \rho)}(x), \quad \quad x \in \mathbb{R}^{n},
$$

we have

$$
H^{*}(t) \leq\left(I_{\alpha} * \tilde{g}_{\varepsilon}\right)^{*}(t), \quad t>0 .
$$

These estimates and (3.53) imply (see also Remark 3.8(ii))

$$
\begin{aligned}
& \int_{B(0, \rho)} \Phi\left(\left|\left(I_{\alpha} * \tilde{g}_{\varepsilon}\right)(x)\right|\right) \mathrm{d} x=\int_{\mathbb{R}^{n}} \Phi(H(x)) \mathrm{d} x \\
= & \int_{0}^{K} \Phi\left(H^{*}(t)\right) \mathrm{d} t \geq \int_{K / 2}^{K} \Phi\left(\varepsilon \cdot G\left(\frac{K}{2}\right)\right) \mathrm{d} t \\
= & \frac{K}{2} \exp \left[a_{2} \exp \left(a_{1} \cdot \varepsilon^{-q /(\gamma+1)} \cdot G\left(\frac{K}{2}\right)^{-q /(\gamma+1)}\right)\right] \rightarrow \infty \text { as } \varepsilon \rightarrow 0_{+} .
\end{aligned}
$$

Moreover,

$$
\left\|\tilde{g}_{\varepsilon}\right\|_{n / \alpha, r, 1 / r^{\prime}, \delta / r}+\left\|\tilde{g}_{\varepsilon}\right\|_{1} \leq 1
$$

for $\varepsilon$ sufficiently small. Therefore, (3.54) contradicts (3.45).

Using the translation $y=x-x_{0}$, the general case $x_{0} \in \mathbb{R}^{n}$ can be reduced to the case $x_{0}=0$.

\section{Remarks 3.11.}

(i) Unfortunately, the method of the proof of Theorem 2.5 does not determine whether in the case $r>q$ the condition $\gamma<-1,(\gamma+1) / q-(\delta+1) / r+1=$ 0 is necessary for the validity of (2.7) provided that $Q \subset \mathbb{R}^{n}$ is open. Indeed, in this case we would need the inequality $(3.52)$ with $\gamma_{1}=\gamma$ (in order to get a contradiction with Lemma 3.2). However, on taking

$$
h(t)=\log ^{-(\gamma+1) / q}(e+|\log t|), \quad t \in(0, K),
$$


we can easily check that such a inequality does not hold.

(ii) Let $Q \subset \mathbb{R}^{n}$ be a measurable set, $K:=|Q|_{n} \in(0, \infty]$. Assume that $p$, $q \in(0, \infty], \lambda, \varepsilon \in \mathbb{R}$ and put

$$
L^{p, q}[\log L]^{\lambda}[\log \log L]^{\varepsilon}(Q):=\left\{u ;\|u\|_{p, q, \lambda, \varepsilon, Q}<\infty\right\}
$$

where

$$
\|u\|_{p, q, \lambda, \varepsilon, Q}:=\left\|u \chi_{Q}\right\|_{p, q, \lambda, \varepsilon}
$$

obviously,

$$
\|u\|_{p, q, \lambda, \varepsilon, Q} \leq\left(\int_{0}^{K}\left[t^{1 / p}(e+|\log t|)^{\lambda} \log ^{\varepsilon}(e+|\log t|) u^{*}(t)\right]^{q} \frac{\mathrm{d} t}{t}\right)^{1 / q}
$$

if $q<\infty$, and

$$
\|u\|_{p, \infty, \lambda, \varepsilon, Q} \leq \sup _{0<t<K} t^{1 / p}(e+|\log t|)^{\lambda} \log ^{\varepsilon}(e+|\log t|) u^{*}(t) .
$$

Then the inequality (2.4) implies that

$$
\|u\|_{\infty, q,-1 / q, \gamma / q, Q} \leq C\|f\|_{p, q_{1}}\left(\|g\|_{p^{\prime}, q_{2}, 1 / r^{\prime}, \delta / r}+\|g\|_{1}\right)
$$

for any $Q \subset \mathbb{R}^{n}$ with $|Q|_{n}=K \in(0, \infty)$. This means that the operator $g \rightarrow f * g$ is continuous from

$$
L^{p^{\prime}, q_{2}}[\log L]^{1 / r^{\prime}}[\log \log L]^{\delta / r} \cap L^{1}\left(\mathbb{R}^{n}\right)
$$

into

$$
L^{\infty, q}[\log L]^{-1 / q}[\log \log L]^{\gamma / q}(Q) .
$$

The inequality (2.5) can be interpreted similarly.

(iii) A particular choice $r=n / \alpha, q=r, \gamma=-r$ and $\delta=0$ in Corollary 2.4 implies that

$$
\exp \left(a_{2} \exp \left[a_{1}\left|I_{\alpha} * g\right|^{n /(n-\alpha)}\right]\right) \in L_{\text {loc }}^{1}\left(\mathbb{R}^{n}\right)
$$

for any $a_{1}, a_{2} \in(0, \infty)$ and for any function $g$ satisfying

$$
g \in L^{n / \alpha}[\log L]^{1-\alpha / n} \cap L^{1}\left(\mathbb{R}^{n}\right) .
$$

Suppose now that $\Omega \subset \mathbb{R}^{n}$ with $|\Omega|_{n} \in(0, \infty)$ and let $g$ be a measurable function on $\mathbb{R}^{n}$ with $\operatorname{supp} g \subset \Omega$. Then the condition (3.55) holds if and only if $g \in L^{n / \alpha}[\log L]^{1-\alpha / n}$. The last relation is in turn equivalent to

$$
\int_{\Omega}\left[|g(x)| \cdot \log ^{1-\alpha / n}(e+|g(x)|)\right]^{n / \alpha} \mathrm{d} x<\infty
$$


(cf. [BR, Corollary 10.2]). Hence we obtain the result mentioned in the third paragraph of the Introduction.

(iv) Let $q, r \in(1, \infty), \alpha \in(0, n), \gamma, \varepsilon \in \mathbb{R}$. Applying the Hardy inequality (1.3) (with $\delta=\varepsilon$ ) instead of (3.1), we are able to show that

$$
\left(\int_{0}^{1}\left[(1+|\log t|)^{\gamma / q}\left(I_{\alpha} * g\right)^{*}(t)\right]^{q} \frac{\mathrm{d} t}{t}\right)^{1 / q} \leq C\left(\|g\|_{n / \alpha, r, \varepsilon / r}+\|g\|_{1}\right)
$$

for all $g \in L^{n / \alpha, r}[\log L]^{\varepsilon / r} \cap L^{1}\left(\mathbb{R}^{n}\right)$ if and only if (cf. (2.3))

$$
\begin{cases}\text { either } & r \leq q, \quad \gamma<-1, \quad \frac{\gamma+1}{q}-\frac{\varepsilon+1}{r}+1 \leq 0, \\ \text { or } & r>q, \quad \gamma<-1, \quad \frac{\gamma+1}{q}-\frac{\varepsilon+1}{r}+1<0,\end{cases}
$$

which gives the single exponential integrability result

$$
\exp \left(a\left|I_{\alpha} * g\right|^{-q /(\gamma+1)}\right) \in L_{\mathrm{loc}}^{1}\left(\mathbb{R}^{n}\right)
$$

for any $g \in L^{n / \alpha, r}[\log L]^{\varepsilon / r} \cap L^{1}\left(\mathbb{R}^{n}\right)$ and any $a \in(0, \infty)$. This is an extension of results of Brézis and Wainger applied to the Riesz potential (cf. [BW, Theorems 2, 3]):

$$
\left(\int_{0}^{1}\left[(1+|\log t|)^{-1}\left(I_{\alpha} * g\right)^{*}(t)\right]^{r} \frac{\mathrm{d} t}{t}\right)^{1 / r} \leq C\left(\|g\|_{n / \alpha, r}+\|g\|_{1}\right),
$$

and

$$
\exp \left(a\left|I_{\alpha} * g\right|^{-r^{\prime}}\right) \in L_{\mathrm{loc}}^{1}\left(\mathbb{R}^{n}\right)
$$

for any $g \in L^{n / \alpha, r} \cap L^{1}\left(\mathbb{R}^{n}\right)$ and any $a \in(0, \infty)$. (On taking $q=r$, $\gamma=-r$ and $\varepsilon=0,(3.59)$ and (3.60) follow from (3.56) and (3.58), respectively.) This approach does not give rise to the double exponential integrability result mentioned above; for this, it appears that the double limiting form of Hardy's inequality (3.1) and the generalized Lorentz-Zygmund spaces $L^{n / \alpha, r}[\log L]^{1 / r^{\prime}}[\log \log L]^{\delta / r}$ are essential. Note that the limiting case $\gamma=-1$ in (3.57) corresponds to the limiting value $\varepsilon=r-1$ in (3.57), which in turn gives rise to the limiting exponent $1 / r^{\prime}$ at the $\log L$-term. Of course, this process can be continued with higher order logarithms producing higher order exponential integrability: thus a convolution on the intersection of generalized Lorentz-Zygmund space $L^{p, q}[\log L]^{\lambda_{1}} \ldots[\log \ldots \log L]^{\lambda_{k}}$ (with 
limiting exponents $\left.\lambda_{1}, \ldots, \lambda_{k-1}\right)$ with the space $L^{1}\left(\mathbb{R}^{n}\right)$ leads to results of the form

$$
\exp \left(a_{k} \exp \left(a_{k-1} \ldots \exp \left(a_{1}\left|I_{\alpha} * g\right|^{-q /(\gamma+1)}\right) \ldots\right)\right) \in L_{\text {loc }}^{1}\left(\mathbb{R}^{n}\right)
$$

(v) The double exponential integrability result can be interpreted in terms of spaces of Sobolev type, giving a result similar to that of Trudinger mentioned in the Introduction. Thus, taking $u \in C_{0}^{\infty}\left(\mathbb{R}^{n}\right)$ and using the estimate

$$
|u(x)| \leq C(n, k) I_{k} *\left(\left|D^{k} u\right|\right)(x), \quad x \in \mathbb{R}^{n},
$$

where $k \in\{1,2, \ldots, n-1\},\left|D^{k} u\right|:=\sum_{|\nu|=k}\left|\left(\partial^{|\nu|} u / \partial x^{\nu}\right)\right|$ (see, e.g., $[\mathrm{Z}$, Remark 2.8.6]), Corollary 2.4 yields:

Let $k \in\{1,2, \ldots, n-1\}$ and let $q, r \in(1, \infty), \gamma, \delta \in \mathbb{R}$ be such that $(2.3)$ holds. Let $Q \subset \mathbb{R}^{n},|Q|_{n}<\infty$ and $a \in(0, \infty)$. Then there exist positive numbers $A=A(n, q, r, k, \gamma, \delta)$ and $M=M\left(a,|Q|_{n}\right)$ such that the inequality

$$
\int_{Q} \exp \left(a \exp \left[A|u(x)|^{-q /(\gamma+1)}\right]\right) \mathrm{d} x \leq M
$$

holds for all $u \in C_{0}^{k}\left(\mathbb{R}^{n}\right)$ satisfying $\left\|\left|D^{k} u\left\|_{n / k, r, 1 / r^{\prime}, \delta / r}+\right\|\right| D^{k} u \mid\right\|_{1} \leq 1$.

Acknowledgement. The work was started when the second author was at the University of Sussex as a research fellow and the third author visited this university. We are grateful for having had this opportunity.

\section{REFERENCES}

[BR] C. Bennett \& K K. Rudnick, On Lorentz-Zygmund spaces, Dissertationes Math. 175 (1980), 1-72.

[BS] C. Bennett $\mathscr{E}$ R. Sharpley, Interpolation of operators, Pure Appl. Math., vol. 129, Academic Press, 1988.

[BW] H. Brézis 85 S. Wainger, A note on limiting cases of Sobolev embeddings and convolution inequalities, Comm. Partial Differential Equations 5 (1980), 773-789.

[HMT] J. A. Hempel, G. R. Morris $\mathcal{E}$ N. S. Trudinger, On the sharpness of a limiting case of the Sobolev imbedding theorem, Bull. Austral. Math. Soc. 3 (1970), 369-373.

[L] G. Lorentz, On the theory of spaces $\Lambda$, Pacific J. Math. 1 (1951), 411-429.

[O] R. O'NeIL, Convolution operators and $L(p, q)$ spaces, Duke Math. J. 30 (1963), 129-142.

[OK] B. Opic $\&$ A. Kufner, Hardy-type inequalities, Pitman Research Notes in Math. Series 219, Longman Sci. \& Tech., Harlow, 1990.

[R] W. Rudin, Real and complex analysis, McGraw-Hill, Inc., 1974.

[S] E. T. SAWYER, Boundedness of classical operators on classical Lorentz spaces, Studia Math. 96 (1990), 145-158. 
[T] N. S. Trudinger, On imbeddings into Orlicz spaces and some applications, J. Math. Mech. 17 (1967), 473-483.

[Z] W. P. Ziemer, Weakly differentiable functions, Graduate Texts in Mathematics, Springer-Verlag, Berlin, 1989.

The research of the second author was supported by the Royal Society of London, the research of the other authors was partially supported by a NATO Collaborative Research Grant, no. CRG 930358.

DAvid E. EDmunds

Centre for Math. Analysis \& Applications

The University of Sussex

Falmer, Brighton BN1 9QH England

E-mail: d.e.edmunds@sussex.ac.uk

Bohumír Opic

Mathematical Institute

Academy of Sciences of the Czech Republic

Žitná 25

11567 Prague 1, Czech Republic

E-mail: opic@earn.cvut.cz

Received: November 29, 1993; revised: July 29, 1994.

\author{
Petr Gurka \\ Department of Mathematics \\ Czech University of Agriculture \\ 16021 Prague 6, Czech Republic \\ E-mail: gurka@tech.vsz.cz
}

